\title{
Consciousness and Topologically Structured Phenomenal Spaces
}

\author{
Robert Prentner ${ }^{1}$
}

\begin{abstract}
There are strong reasons to believe that our conscious inner life is structured, suggested both by introspection as well as scientific psychology. One of the most salient structural characteristics of conscious experiences is known as unity of consciousness. In this contribution, we wish to demonstrate how features of experience that pertain to the unity of consciousness could be made precise in terms of mathematical relations that hold between phenomenal objects.
\end{abstract}

Based on phenomenological considerations, we first outline three such features. These are (i) environmental embedding, (ii) the mutual constraint between local and global representations, and (iii) a top-down process of object formation in consciousness. We then introduce a formal model based on the notion of phenomenal space, defined in terms of a set of quasi-elementary and extended entities. We describe the structure of phenomenal space by appealing to mereological and topological concepts, and we outline a projector-based calculus to account for the idea that the structure of phenomenal space is ultimately dynamical.

Using the above concepts, one could approach the mind-matter problem by relating environmentally embedded agents to the topologically well-defined objects that result from decompositions of phenomenal space. We conclude our discussion by putting it into the context of some recent theoretical questions that appear in cognitive science and consciousness studies. We opt for the possibility to regard the phenomenon of consciousness not in terms of a singular transition that happens between "brain" and "mind" but rather in terms of a series of transitions between structured layers of experience.

1 robert.prentner@uci.edu

This is the author's accepted manuscript. Please cite the published version:

R. Prentner: Consciousness and Topologically Structured Spaces, Consciousness and Cognition, 70: 25-38, 2019.

(C) 2019. This manuscript version is made available under the CC-BY-NC-ND 4.0

license http://creativecommons.org/licenses/by-nc-nd/4.0/ 
Keywords: mathematical phenomenology; preconscious background; unity thesis; composition; decomposition; quasi-analysis; phenomenal space; mereology; topology; boundaries

\section{The Unity of Consciousness as Structural Property}

In this article, we take "consciousness" to refer to a process that leads to experience of organisms. ${ }^{2}$ While ontologically neutral, our approach emphasizes that experience (and thus the process leading to it) is structured. The unity of consciousness is often thought to be one of the most salient features that distinguishes consciousness from other natural processes: When consciously perceiving ourselves and the environment, we are not separately aware of different features of our experience (say a color and, separately, the texture of a surface) but experience them together - forming an "experiential whole", with colored surfaces interwoven with other perceptions, a sense of self, thoughts and memories. This is surprising insofar as the corresponding neural processes are often localized in different areas of the brain, are processing different kinds of (sensory) input and are regulating different kinds of (behavioral) output. The phenomenological finding - if correct - thus defines a serious challenge for theories that equate consciousness with the state of some particular neuronal assembly or with other, more abstract characterizations of the brain. What makes this even more interesting is the fact that it affects both scientific approaches to consciousness (e.g. Revonsuo 1999, Engel \& Singer 2001, Tononi 2008, Oizumi et al. 2014) as well as philosophical ones (e.g. Searle 2000, Bayne \& Chalmers 2003, Bayne 2010).

On a dualist reading, the unity of consciousness is not in need of explanation. In dualism, "unity" simply reflects the "essence" or "nature" of the mind (as opposed to matter). Since dualism, however, is a philosophical doctrine that most researchers in the field are keen to reject, one needs to account for unity in a

\footnotetext{
2 The terms "consciousness" and "experience" are often used interchangeably. More precisely, throughout this article, experience - which might be described in terms of a state - should be conceived of as "resulting from" consciousness - which refers to a process. An example in case is (conscious) perception, conceived of as process leading to the experience as of shapes, colors etc. We shall sometimes also use the term "conscious experience" to emphasize that the experience in question is specifically related to consciousness. A large part of this article is devoted to construct a formal architecture to make some of these ideas more precise.
} 
different way. Explaining various experiential phenomena related to the notion of unity is therefore one of the major desiderata for any (non-dualist) science of consciousness.

In this context it is important to clarify the role of introspection. On the surface and according to the Cartesian tradition in cognitive science, introspection is sometimes understood as "observation" of inner mental states. A detailed account of introspection is then often equated with a phenomenological analysis. We believe this conflation of introspection and phenomenology is unfortunate, however, because it misses the idea that phenomenology is not simply concerned with some given "raw material" of perception. Instead phenomenology, as conceived by Edmund Husserl more than a century ago, has always been concerned with making intelligible the "structural invariants" that underlie experience - as opposed to the way we, as individuals and members of some biological species, verbally express it, remember it or merely talk about it. The idea of an "inner mental life" which could be "observed" in introspection is a confabulation that derives from a much subtler process. Moreover, mathematics seems to be the best tool we have for inter-subjectively establishing the structural invariants of conscious experience. Therefore, the following article could be thought of as contributing to a "mathematized phenomenology" (Yoshimi 2007) that is open to but also constrains findings from cognitive science.

According to one particular view, consciousness correlates to the complexity of bio-physical information processing networks (e.g. Tononi 2008). More in line with traditional phenomenology, such networks should be regarded as "realizations" by which consciousness gets objectified, rather than referring to "correlates" that are (functionally or physically) identical to it. It follows that the networks in question should exhibit a structure sufficiently related to the structure of experience - given perhaps in terms of an isomorphism between mathematical representations but perhaps also in a more sophisticated fashion ${ }^{3}$. Therefore, the progress of biological theories also depends on our ability to give a formal (mathematical) but also phenomenologically adequate description of the structure of experience. In this article, we shall not be concerned much with the particular bio-physical mechanisms. Instead, we shall focus on some of the general structural properties such mechanisms ought to exhibit.

\footnotetext{
${ }^{3}$ For example, in terms of a supervenience relation between state space descriptions (Yoshimi 2011); in terms of topological invariants between conscious and unconscious processes (Sieroka 2015); or in terms of category theory (Tsuchiya et al. 2016).
} 
The unity of consciousness is a problematic notion that needs serious unpacking. In section 2 of this paper, we shall start by giving an outline of some characteristics of experience that are often thought to stand for (or relate to) "unity" in some particular sense. We shall introduce three ideas based on phenomenological and philosophical considerations. We assume that conscious experience is structured along multiple interdependent layers: First, experience is always given against a "preconscious background." Preconscious states, related to perception-action couplings that are themselves not explicitly represented by the organism, specify the basis for the object-content of experiences. An organism's "Umwelt" (von Uexkull 1926) or primitive "feeling states" (Damasio \& Carvalho 2013) are possible candidates for supporting preconscious background states. Also "affordances", understood as relations between abilities of organism and environmental features (Chemero 2003), or the totality of an organism's "sensorimotor contingencies" (O’Regan \& Noë 2001) might express empirical correlates of preconscious states. Second, unity could be understood in terms of a relationship between system-wholes and its parts which is one of interdependence, unlike purely "building block approaches to consciousness" (Searle 2000) that postulate conscious experience to be the sum of well-defined and separable phenomenal parts but also unlike "single state conceptions" (Wiese 2016) that postulate a purely holistic framework to account for conscious experience. Third, consciousness is often described as having a "transcendental character" (Kant 1998); this implies that consciousness dynamically grounds our ability to perceive a differentiated phenomenal field composed of well-defined and unified objects instead of experiencing a phenomenal "pulp" of undifferentiated "what-it-is-like-ness" (Nagel 1974).

In section 3, we shall explicate these ideas in terms of mathematical concepts. The basic "elements" of experience are argued to refer to extended entities rather than to non-extended and duration-less "points." Based on this assumption, we introduce the notion of "phenomenal space." We thereby apply concepts from mereology, the formal study of part-whole relations (Simons 1987) and topology, the study of pre-metric relations. Our approach is to combine these concepts (Smith 1996, Casati \& Varzi 1999) to account for the structure of phenomenal space spanned by extended elements.

The way phenomenal space is partitioned into objects is mirrored by the boundaries which are imposed on collections (sets) of its elements. Imposing boundaries is assumed to be an endogenous process, and to account for this we 
shall introduce an operator-based representation. A well-known example pertains to vision where objects in the visual field are experienced as spatially structured and bounded from each other; however, our analysis is believed to generalize to other sensory modalities as well and perhaps even to non-sensory experiences such as emotions, insofar as these involve the formation of object-content, or modes of cognition, insofar as these have a distinct "cognitive phenomenology" to them (Pitt 2004).

Finally, in section 4 we shall summarize the above and discuss how our mathematical descriptions relate to the phenomenological characterization of experience. We also wish to briefly discuss the relation of our model to current issues in cognitive science and consciousness studies.

\section{Concepts for Mathematical Phenomenology}

\subsection{Toward a Formalism to Express Unity}

The possibility of scientifically studying the phenomenology of consciousness rests on the availability of robust formal models that account for the possible structure of experience. Assuming that structure is often concealed and not readily given to introspection (if understood in terms of "inner observation"), exposing this hidden structure of experience requires careful phenomenological analyses. In turn, communicability between different researchers becomes crucial - something akin to a notational system of the mind or a formal semantics for psychology.

One particular approach is the analysis given by Marbach who proposed, and then also related, different kinds of "intentional implications and modification" (Marbach 2009, p. 63) to account for various types of "conscious acts" such as perception, recall from memory, or imaginary thinking. Crucially, this included the statement that every conscious act involving an intentional object " $x$ " is grounded in a subjective unity " $i$ " against the background of a presentation (“( $P R E)$ ”) of the environmental surrounding "s"; written semi-formally:

$$
i_{\overline{(P R E) S}} \ldots \mid x
$$

Here, "presentation" refers to an immediately given (implicit) relation between the perceptual agent and its environment. This should be distinguished from an 
(explicit) "representation," which consists in the agent's directedness "..." towards intentional objects (in the modes of, e.g., perception, recall, or imaginary). In other words, intentional conscious thought is understood as "re-presentation" in the literal sense, as re-lived presentation of something absent, whereas a "presentation of environment" amounts to its directly being perceived as being present. Subjectively, this corresponds to the feeling of being present here-andnow in an external world around the self (Revonsuo 2006).

How could the above phenomenological formalism be applied to empirical research? One example is discussed by Marbach (2009), when he cites research by Kosslyn et al. (2001) into the foundations of mental imagery and in particular their claim that "imagery, unlike perception, does not require low-level organizational processing, whereas perception, unlike imagery, does not require us to activate information in memory" (Kosslyn et al. 2001, p. 636). However, according to Marbach, there are further differences (encoded by the "intentional implications and modifications" in his formalism) that would need to be distinguished explicitly in order for the experimental target phenomena to count as phenomenologically adequate instances of perception or imagery. In other words, while one might find (non-)overlap between physiological correlates of experiences, these findings are fundamentally incomplete and need to be supplemented with phenomenologically-informed knowledge. A similar example pertains to the increasingly-popularized idea that neuroscience could be used to "read" the mind of test-subjects. While it is certainly true that fMRI provides one with insights into the physiological correlates of the human mind (this is a truism), one needs to assume some sort of "compositionality of thought" to sustain a more comprehensive claim such as "mind-reading."

Some empirical studies seem to suggest compositionality for particular cases (Wang et al. 2017). Are there any insights from phenomenology that shed light on the nature of compositionality more generally? Structurally, there is an important commonality between the different modes of experience discussed by Marbach: It is their embedding in an unifying structure imposed on the presentation of the organism's surroundings, which is thought to accompany every conscious experience. It is this structural property of conscious experience which shall be further characterized in the present work. Instead of assuming a brute form of compositionality where it is simply assumed that local phenomenology jointly constitutes global phenomenology comprehensively, we propose a model of co-constitution. 
That conscious experience always needs to be conceived of with reference to an overarching mental "scheme of things" finds expression in a "unity thesis", such as the one proposed by Bayne \& Chalmers (2003). In it, the authors state that "necessarily, any set of conscious states of a subject at a time is unified" (Bayne \& Chalmers, 2003, p. 24). Limiting this to phenomenal states ${ }^{4}$, we could express this in a slightly modified version in a more formal way:

$$
\forall x\left(P(x) \rightarrow \exists \zeta\left(P(\zeta) \wedge \zeta=F\left(x, \phi_{1}, \phi_{2}, \ldots \phi_{n}\right) \wedge S(x, \zeta)\right)\right)
$$

The formula says that for every phenomenal $(P)$ state $x$ there exists one "global" phenomenal state $\zeta$ which is a "fusion" $(F)$ of all momentarily present phenomenal states $\left\{x, \phi_{1}, \ldots, \phi_{n}\right\}$ but also "subsumes" $(S)$ it, implying that "on its own" and irrespective of this embedding, $x$ were not a phenomenal state. By this alone, not much has yet been said about the details of fusions or the supposedly subsumptive structure of experience. Still, it implies that a mere "sum" of elements $x$ and $\phi_{i}$ is not enough to account for the phenomenology of consciousness. Throughout this article, two complementary processes are assumed to take place in experiencing systems: one that makes intelligible how different elements constitute unitary experience, and one that makes intelligible how this experience could be a structured one, i.e., how it could be conceived of as being decomposable into distinct parts. This implies a reciprocity underlying the dynamics of consciousness.

If we do not consider this interdependency, we will be faced with explanatory puzzles. One such puzzle pertains to the project of finding "neural correlates of consciousness" (NCCs; Metzinger 2000, Hohwy 2009, Koch et al. 2016): If one could not explain in which sense a local neuronal state, correlating to a consciously perceived content, is subsumed under a global neuronal state, itself correlating to "being conscious" as opposed to "being unconscious" (e.g. being awake, sleeping, or dreaming), it would stay unintelligible why the particular content appears (to be unified) at all.

\footnotetext{
${ }^{4}$ We mainly restrict ourselves to the alleged structural unity of the phenomenal field (cf. Bayne 2010). We thereby doubt whether one could sensibly speak of "co-conscious" (Bayne \& Chalmers 2003) states, if consciousness were understood in terms of a "building-block" model (Searle 2000) in which the overall conscious state is the result of particular mental sub-states which could be regarded conscious states themselves. Instead, we are favoring a semi-global model where wholes and parts are undergoing a mutually constrained relationship.
} 
Conversely, if one looked at consciousness from a perspective which only recognized the subsumptive structure of experience, it would be impossible to explain why experience is partitioned the way it is. This would have enormous impact on empirical research: Scientists would not be able to give answers to the question why this kind of biological activity is correlated to that kind of experience, which is arguably the most important desideratum for a neuroscience of consciousness.

There is also a third idea related to the unity of consciousness which we wish to discuss at this point, and which could aptly be named the "Kantian idea." Kant's (1998) theory of the transcendental unity of consciousness that he developed in his Critique of Pure Reason is notoriously difficult to understand. We do not wish to enter into the field of Kant exegesis at this point, but we wish to emphasize one salient feature of the Kantian conception of unity. Accordingly, it refers to a property of experience that every conscious being imposes on the raw sensory material that has been structured in terms of space and time. In fact, it is fair to say that, for a transcendentalist, consciousness is not something that happens "in" space and time or that represents objective space-time categories. Rather, space and time are thought of as the "forms of" experience out of which perceived objects emerge (Fig. 1). Recently, remarkably similar hypotheses have been proposed, according to which biological activity shapes the perception of space (Therekov \& O’Regan 2016, Buzsáki \& Llinas 2017) or where spacetime emerges as "error correcting code" from the activity of agents that embody predictions of the fitness consequences of their actions (Fields et al. 2017). In these proposals, it is assumed that space and time as perceived are possibly quite different to the concepts of (physical) space and time and do not mirror some object-structure which (supposedly) exists independently.

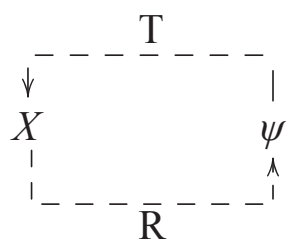

Figure 1: Circular structure and transcendental unity of consciousness: $X$ denotes the physical state of the environment; $\psi$ a state description of conscious experience. Arrows represent grounding relations that are responsible for the unity of the grounded object. Transcendentalists believe in the grounding role of consciousness for the perception of unified 
objects (upper arrow); strong representationalists believe that the unity of consciousness stems from the "real" properties of represented physical objects (lower arrow).

In our mathematical model, a transcendentalist interpretation arises naturally. However, one might stay agnostic on its metaphysical implications but simply note that, following phenomenology and critical philosophy, the dynamics of consciousness underlies the appearance of objects as unified. We therefore need to account for the tendency of conscious beings to perceive objects, boundaries or substances, when all there is are interactions between a living entity and its environment. What this translates to is a "top-down" approach to objectperception which views objects not as "givens" but as constructions.

We have identified three conceptions that pertain to conscious experience and are often thought of as being related to the unity of consciousness: (i) that conscious experience is supported by an organism's environmental embedding, (ii) that it is situated in a mutually constrained process which involves the fusion of (structured) elements into a sum that constrains its parts, and (iii) that it results in perceiving distinct and unified objects. In order to explicate a mathematical structure which accounts for these features, we first need to state the elements entering into the corresponding relations.

\subsection{Composition and Elements}

Trying to mathematically represent the structure of experience starts by asking whether or not experience is constituted by certain (idealized) "elements." Either this is literally the case, then it makes sense to carve up experience into components; or, if not, explicating the structure of experience implies the need of a "quasi-analysis" (Carnap 1967): a theoretical representation of experience in terms of a structure that is defined on hypothetical (= non-empirical) elements. The historically most pertinent example pertains to Gestalt-psychology. Its proponents assumed that experience is not constituted by "Wundtian" elements, that is, by indivisible and point-like relata of psycho-physical relations, but that experience is always given in terms of an extended shape - a "Gestalt." Accordingly, any formal representation of experience that is consistent with Gestalt-psychology would need to introduce quasi-elements of experience and show how they could give rise to Gestalt structures. Furthermore, these elements would not - per assumption - have an empirical counterpart and could not be investigated directly. 
A more contemporary example regards the conflict between view-based approaches (e.g. Poggio \& Edelman 1990) in computational vision compared to an element-approach such as Biedermann's (1987) "recognition-by-components"theory. In Biedermann's theory a modest-sized set of elementary and viewpointinvariant shapes, called "geons", underlies object recognition. Examples for geons are blocks, cylinders or cones. A critique of this theory concerns two related questions: (i) whether the list of elementary shapes is complete (there seems to be no a priori reason for this), and (ii) whether natural images can in fact be modeled in terms of compositions of geons (many natural objects such as the Human ear are difficult, perhaps impossible, to model with geons). A possible reaction to these difficulties is to doubt the assumption that recognition of natural images could be understood in terms of a composition of fixed elementary shapes. The dispute between view-based approaches and a geon-based theory thus conceptually mirrors the Gestalt-theoretical opposition to Wundtian elements. To summarize, stating distinct phenomenal elements seems difficult for the following two reasons:

1) While we could easily conceive of the elementary objects of the biological substrate (single cells or neuronal assemblies), the phenomenology of consciousness is such that it seems composed of an indefinite number of (phenomenal) parts. An example in case pertains to the experience of continua. The continuum could be defined as "an entity that could continually be divided without ever arriving at some final, indivisible elements." (Weyl 1988). While it is true that we could reconstruct the concept of the continuum in terms of a set of indivisible elements (i.e., in terms of a set of points), this reconstruction would not adequately capture the experience of continua: Each part of a continuum is always experienced itself as being structured; logically, no representation in terms of points accounts for this. The idea of elementary qualia that combine to experiences is problematic for similar reasons.

2) If, by contrast, an elementary analysis of consciousness were indeed possible, this would immediately raise the question why the phenomenology of consciousness does not correspond to a structured set of elementary (pointlike) experiences but rather to a unified experiential whole. In addition, all attempts to derive a one-to-one correspondence between the supposed "elements of the mind" and the "elements of the brain" (like the cell or the neuronal assembly) have so far been futile. 
The above should not be taken as fact about (im-)possibilities of representation, but as (educated) guess about a good starting point. While the prospects might seem dim for an elementary analysis of experience into empirically existent phenomenal micro-objects (along the lines of an elementary analysis of matter into atoms), we should not give up the project of quasi-analysis. The quasianalysis of experience, as we intend to give in this article, should simultaneously account for the apparent indivisibility of experience and the requirement of analyzability into well-defined parts in order to do science.

Quasi-elements are hypothesized not to resemble point-like instances. This reflects phenomenological (Gestalt-psychological) considerations but also the hypothesis according to which quasi-elements correlate to processes that relate sensations and actions to keep an organism viable. Such processes are extended in space and time and likely involve information transfer, internal decision mechanisms and target selection, all of which could in principle be conceived of as being de-localized and non-instantaneous.

\section{Formalism}

\subsection{Phenomenal Space and Decomposition}

We start by introducing the concept of a "phenomenal space" and then later the notions of "part-whole relation" and "topological structure." Throughout the mathematical sciences, the concept of a space, or a sufficiently structured set of elements, is widespread. The best know example probably is a Euclidean metric space. However, phenomenal space differs in some important respects. First, the elements that compose phenomenal space are not necessarily isomorphic to empirically existing (idealized) point-like particles; as we have suggested before, phenomenal space is most likely composed of quasi-elements that themselves allow for further analysis and are extended. If they satisfy a similarity criterion, two quasi-elements are said to "overlap" (as defined more rigorously in section 3.2.), amounting to the most primitive relation underlying the (compositional) structure of phenomenal space. Second, however, this structure is not necessarily metric. Although this might be so in some particular cases (e.g., in the case of color experiences), in others it is doubtful (e.g., in the case of emotional experience or attributions of meaningfulness). Still, we would like to assume that phenomenal space is structured. If we demand it to be measurable, phenomenal 
space should possess a structure known as sigma-algebra ${ }^{5}$. For a geometrical appreciation, it is most useful to think in terms of topology. Intuitively, the topology on a set of objects defines their neighborhood-relation or their connectedness; in the discrete case, it is straightforward to construct a link between topological spaces and measurability ${ }^{6}$.

In any experiencing organism, the currently ${ }^{7}$ realized configuration of quasielements is said to form the "preconscious background." We furthermore wish to distinguish between the preconscious background and the experienced objects themselves. Only the latter are hypothesized to contribute to the currently experienced contents ${ }^{8}$ of consciousness. In our model, phenomenal space is compositional with respect to quasi-elements, but it is decompositional with respect to the object-contents of conscious experience. The former implies that the set of quasi-elements generically combines to phenomenal space. The same, however, is not true for the objects of experience; such objects relate to phenomenal space in the sense that they result from the way how phenomenal space is partitioned.

The following is intended to give an illustration of the difference between compositional and decompositional aspects using the simple example of probability distributions, where "decomposition" corresponds to marginalization and "composition" corresponds to inferring a joint probability from marginal ones:

\footnotetext{
${ }^{5}$ A sigma algebra is a collection of subsets ("events") of some set that fulfils certain axioms (see, e.g., Tao 2011). The set of elements together with the sigma-algebra defines a "measurable space." We agree with Hoffman et al. (2015) that measurability is likely the most basic "condition of possibility" for any scientific study of consciousness. It is possible in this case to define a measure that assigns those events a certain number. "Co-measurability" of complexes of quasi-elements perhaps is a good proxy for what Carnap (1967) conceived of as (strong) similarity relation between elementary experiences.

${ }^{6}$ Topology naturally "picks out" all open (closed) sets defined on $X$. Given any topological space $X$, the collection of all Borel-sets (defined by union, intersection and complement of open sets) on this space defines a sigma-algebra (cf. Tao 2011).

7 To scholars familiar with Husserl's discussion of time consciousness this immediately raises the question of how to treat "protention", "retention", or more generally: the temporal extendedness of experience. One possibility would be to generalize the notion of phenomenal space to the concept of a "phenomenal spacetime."

8 There is an important question to what extend intentional objects exhaust experiential content, or, in other words, whether qualitative character could be accounted for fully in terms of "object-content." This translates into the question whether all representations of phenomenal experience (e.g. color-experiences) are treatable using the same or a similar model as proposed in this article. I thank one referee to make me aware of this.
} 
Let $x$ and $y$ stand for two quantities which could each take two distinct values $\left\{x_{1}, x_{2}\right\}$ and $\left\{y_{1}, y_{2}\right\}$. The joint probability $p\left(x_{i}, y_{j}\right)$ is the probability to simultaneously find the variables $x_{i}$ and $y_{j}$. In total, there are $2 \times 2=4$ different possible values $p\left(x_{i}, y_{j}\right)$. A concrete example in case would be $p\left(x_{1}, y_{1}\right)=0, p\left(x_{1}, y_{2}\right)=$ $\frac{1}{2}, p\left(x_{2}, y_{1}\right)=\frac{1}{2}$ and $p\left(x_{2}, y_{2}\right)=0$. What is the probability to find $x_{1}$ irrespective of the $y_{j}$ ? This so-called "marginal probability" is given as $p\left(x_{1}\right)=p\left(x_{1}, y_{1}\right)+p\left(x_{1}, y_{2}\right)=\frac{1}{2}$. Analogously, $\quad p\left(x_{2}\right)=p\left(x_{2}, y_{1}\right)+p\left(x_{2}, y_{2}\right)=\frac{1}{2}, \quad p\left(y_{1}\right)=$ $p\left(x_{1}, y_{1}\right)+p\left(x_{2}, y_{1}\right)=\frac{1}{2}$ and $p\left(y_{2}\right)=p\left(x_{1}, y_{2}\right)+p\left(x_{2}, y_{2}\right)=\frac{1}{2}$.

It is easy to verify that the joint probability $p\left(x_{i}, y_{j}\right)$ is not equal to the product of marginal probabilities $p\left(x_{i}\right) p\left(y_{j}\right)$, even though the composed space is defined by the product of $x$ and $y ; S_{\text {tot }}=$ $X \times Y$. In general, it is not possible to fully specify the function $p$ knowing only its marginal values. Conversely, however, if the joint probability on the total space is given, marginal probabilities could be calculated from there. Mathematically, probabilities are closely related to the structure of that space (i.e., $p$ is defined as function on a measurable space, which satisfies certain properties); this structure defines the "events" which could be assigned a probability in the first place.

Compositional and decompositional aspects are ("mereological"; Simons 1987) concepts which pertain to an object's status as part or whole. On a compositional reading the main question is how distinct parts (quasi-elements) combine into a single entity (phenomenal space); on a decompositional reading the main question is how parts (object-contents) could be regarded as abstractions or "results" of a (perhaps biologically-realized) activity that partitions phenomenal space. A compositional approach regards phenomenal space in terms of a collection of phenomenal objects, while a decompositional approach tries to make 
intelligible why we perceive distinct objects at all ${ }^{9}$. Representing the structure of experience, so our claim, requires an understanding of both compositional as well as decompositional principles.

\subsection{Topology}

We wish to characterize the structure of phenomenal space with the help of topology under the constraint that the "points" in space are extended entities. A topological space is given by a set of elements and some neighborhood relation that holds between these elements. Whether something counts as topology on a particular set $X$ could be decided by whether there exists a corresponding closure operator, cl: $\wp(X) \rightarrow \wp(X)$, that creates a closure $\operatorname{cl}(A)$ for every subset $A$ of $X$ and satisfies the following axioms (Kuratwoski 1966):

$$
\begin{gathered}
\operatorname{cl}(\varnothing)=\varnothing, \\
A \subseteq \operatorname{cl}(A), \\
\operatorname{cl}(A \cup B)=\operatorname{cl}(A) \cup \operatorname{cl}(B), \\
\operatorname{cl}(\operatorname{cl}(A))=\operatorname{cl}(A)
\end{gathered}
$$

A set is closed iff all of its points as well as all possible limit points (intuitively, all points that are "near" the set) are contained in it. This imposes an intuitive notion of "structure" on the collections of points in $X$.

To also account for the assumption that the elements which compose phenomenal space are not just unstructured points but extended quasi-elements, we introduce mereotopology (Smith 1996, Casati \& Varzi 1999) which comprises concepts from topology and concepts from mereology. ${ }^{10}$ As primitive (mereological) relation of similarity we choose "overlap"11 between quasi-elements, from which we derive the concepts of "parthood" and "connection." We shall then show how these lead to the concept of (pre-) closure, and later, via the concept of "boundary", to the

\footnotetext{
${ }^{9} \mathrm{~A}$ decompositional approach is a possible candidate to solve some issues in consciousness studies such as the so-called "combination problem" (Seager 1995, Goff 2006, Chalmers 2014).

${ }_{10}$ Equivalently, one could work with point-free topology. Mathematically, this is perhaps more standard. From a pragmatic standpoint, however, mereology has the advantage that it seems to relate more intuitively to phenomenology, because mereological relations could be readily interpreted as representing the relations which figure in experience (for example, different experiences - extended phenomenological complexes - are overlapping iff they could be associated with each other).

11 "Overlap" is taken to refer to a weak similarity relation, that is, a symmetric relationship that is not yet one between measurable events
} 
structure of phenomenal space. Overlap relates various "mental objects" as being weakly similar; such objects could refer to quasi-elements of phenomenal space, arbitrary collections of quasi-elements as they appear in aggregations or in (possibly nested) systems of quasi-elements, or intentional objects.

If any mental object $x$ overlaps with some other mental object $y$, we write this as:

$$
x \circ y .
$$

Given overlap, one could derive a parthood relation, which holds iff all elements that overlap $x$ also overlap $y$ (but not necessarily vice versa: whereas overlap is symmetric, parthood " $<$ " is usually thought to be anti-symmetric, cf. Simons 1987):

$$
x<y \Leftrightarrow \forall z(z \circ x \rightarrow z \circ y) .
$$

Alternatively, one could also define overlap in terms of the parthood relation:

$$
x \circ y \Leftrightarrow \exists z(z<x \wedge z<y) .
$$

To relate mereology to topology, one could introduce a "connection axiom", according to which any two objects, $x, y$, are (mereologically) connected iff there exists a third objects $z$ that overlaps with both $x$ and $y$ such that each of its parts overlaps with $x$ or with $y$ (Whitehead 1919; par. 29):

$$
\forall x y(C(x, y) \Leftrightarrow \exists z(z \circ x \wedge z \circ y \wedge \forall w(w<z \rightarrow w \circ x \vee w \circ y)))
$$

To intuitively see why Eq. (7) accounts for connection, consider two spatially extended regions, $x$ and $y$, that overlap with a third region $z$ (Fig. 2). If every point in $z$ were in $x$ or $y$, then $x$ and $y$ would connect via $z$. 


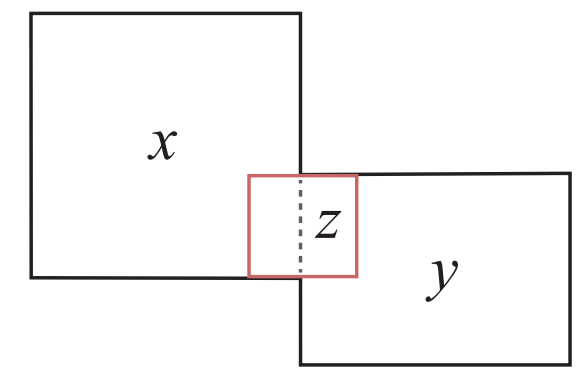

Figure 2: Graphical illustration of the connection axiom using spatial objects (regions).

The purpose behind the connection axiom is to introduce a notion of connectedness that is defined in terms of mereological relations between objects which do not resemble points but stand for extended quasi-elements. To prepare for the transition from mereology to mereotopology, we define a set of objects $A \subseteq X$ together with an operator that acts on phenomenal space:

$$
\begin{gathered}
A=\left\{x_{1}, x_{2}, \ldots x_{n}\right\}, \\
\operatorname{pc}(A)=\left\{z \mid z<A \vee C\left(z, x_{1}\right) \vee C\left(z, x_{2}\right) \vee \ldots \vee C\left(z, x_{n}\right)\right\} .
\end{gathered}
$$

In other words, $\operatorname{pc}(A)$ is the union of objects that are parts of $A$ and mereologically connect to them. It straightforwardly follows that:

$$
\begin{aligned}
& \operatorname{pc}(\varnothing)=\varnothing, \\
& A \subseteq \operatorname{pc}(A) .
\end{aligned}
$$

The operator pc thus satisfies the first two Kuratowski-axioms, Eqs. (3a,b). One could also show that it satisfies the third axiom stated in Eq. (3c):

$$
\begin{gathered}
A=\left\{x_{1}, x_{2}, \ldots, x_{n}\right\} \text { and } B=\left\{y_{1}, y_{2}, \ldots, y_{m}\right\}, \\
\operatorname{pc}(A \cup B)=\left\{z \mid z<A \vee z<B \vee C\left(z, x_{1}\right) \vee \ldots \vee C\left(z, x_{n}\right) \vee C\left(z, y_{1}\right) \vee \ldots \vee C\left(z, y_{m}\right)\right\},(10 b) \\
=\operatorname{pc}(A) \cup \operatorname{pc}(B) .
\end{gathered}
$$

It does not, however, satisfy the last axiom given in Eq. (3d); pc need not be idempotent. (Not everything that mereologically connects to $\mathrm{pc}(A)$ also connects to $A$.) Because of this, we call pc a pre-closure operator. Thus, by introducing the concept of mereological connectedness, it becomes intelligible how extended 
(non-point-like) objects could be said to constitute a (pre-topologically) structured space. One still needs to show how to get to topological structure proper. ${ }^{12}$

\subsection{Boundaries and Objects}

To account for the rich structure of conscious experience, a representation of phenomenal space as pre-topologically structured set is insufficient. A pretopology that is derived from the notion of mereological overlap, as defined in Eq. (8b), is, for example, not enough to capture the idea of bounded objects in perception: If we press together two fingers, we perceive this as two distinct objects that are in contact with each other ("they would connect but not overlap") and not as a single entity. We thus wish to enrich our calculus.

Form Eq. (7) it follows that objects which overlap also connect:

$$
x \circ y \rightarrow C(x, y) \text {. }
$$

Conversely, objects that are disconnected are disjoint:

$$
\neg C(x, y) \rightarrow x<y .
$$

However, this still leaves open the possibility that objects are connected but do not overlap, in which case we say that they connect a boundary:

$$
B(x, z) \Leftrightarrow C(x, z) \wedge x \imath z
$$

To illustrate this, again look at Fig. 2 and assume that the object $z$ collapses into the dashed line which now marks the boundary between the regions $x$ and $y$. If we require that every part of $z$ either belongs to $x$ or $y$ (but not to both),

\footnotetext{
12 There are several strategies to get to topology proper (cf. Casati \& Varzi 1999). A first would solve this by an additional axiom which postulates that any two mereologically connected objects are also topologically connected. Another one is to further restrict the construction of Eq. (8b) in order to satisfy idempotence. This is our strategy and shall be explicated in section 3.3 by introducing the concept of a boundary. There is no purely logical reason to favor one approach over the other. However, an axiomatization that proceeds from overlap between extended quasi-elements and introduces boundaries in terms of additional structural features seems most natural considering the "transcendentalist" idea according to which mind imposes object-hood and boundaries on the preconscious background.
} 
then, according to Eq. (7), $x$ and $y$ would connect but-not overlap. More intuitively, one could say that these objects only appear connected, but there exists some "thing in between" which separates them - a boundary. More, generally, following Smith (1996) and Casati \& Varzi (1999), it is possible to show that mereologically structured spaces equipped with boundaries satisfy closure-axioms and thus define a topology on phenomenal space.

We wish to illustrate this with a toy model, which captures the idea that boundaries induce additional (topological) structure on a set of extended elements (Fig. 3): Conceive of a set of nodes $\xi$ (corresponding to quasi-elements forming the preconscious background) and edges (corresponding to their mutual relation), which together form a graph $G_{\zeta}=(\xi, \circ)$. Any two adjacent nodes are said to "overlap", except if one is (part of) a boundary; and they are said to be "connected" iff there exists some intermediate node that overlaps both. So, for example, adjacent blue nodes overlap, but blue and white nodes merely connect. If for a set $A \subseteq \zeta$, a boundary is definable by those nodes that separate them fro a set of non-overlapping nodes which are part of $\bar{A}=\zeta \backslash \operatorname{cl}(A)$, such a boundary structure picks out a well-defined region in space.

This construction defines a (conceivably simple) topological structure on a graph of extended elements. To see this, we could generalize Eqs. (7) and (12) to relations that hold between objects $y$ and collections of objects, $A$ :

$$
\begin{gathered}
C(y, A) \Leftrightarrow \exists x(x<A \rightarrow x \circ y)), \\
B(y, A) \Leftrightarrow \forall x(x<A \rightarrow C(x, y)) .
\end{gathered}
$$

We could then define:

$$
\operatorname{cl}(A)=\{z \mid C(z, A) \vee B(z, A)\}
$$

which says that $\operatorname{cl}(A)$ includes all objects that are connected to $A^{13}$. In other words, $\operatorname{cl}(A)$ picks out the collection of objects that "lie within" the boundary of $A$. It also follows that distinct objects $\operatorname{cl}(A)$ and $\operatorname{cl}(B)$ could intersect only in their boundary (if at all); their interior parts are disjoint.

${ }^{13}$ In a more formal treatment, one would have to introduce limit-constructions in order to show that closure is satisfied in pathological cases. 


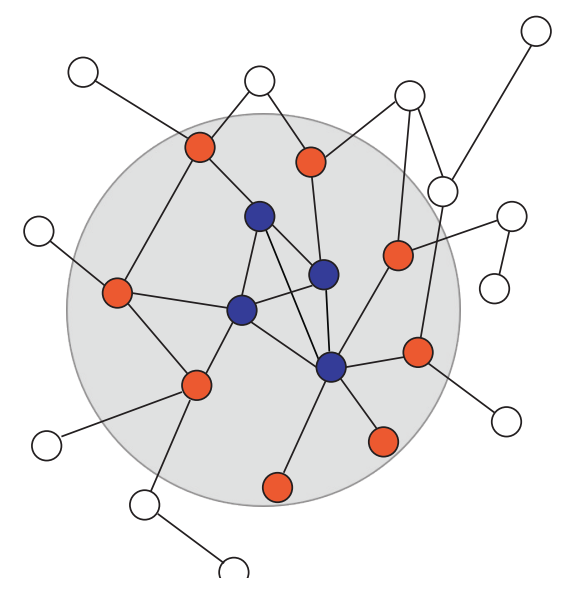

Figure 3: Boundaries defined on the graph $G_{\zeta}$; nodes correspond to quasi-elements of the preconscious background. Any two nodes are "connected" if there exists at most one intermediate node and if they are not separated by a boundary. Red nodes thus connect both with white and blue nodes but do not overlap. The set of red nodes acts as a boundary and specifies a topology on the total set of elements. Such boundaries shield internal from external states and pick out well-defined regions in space (shaded area).

\subsection{Projectors}

In the previous two subsections, we applied concepts from mereotopology. We now wish to go one step further. To account for the top-down character of consciousness, we postulate that topology results from an endogenous dynamics that "sculpts" phenomenal space and thus "generates" the objects of experience. We suggest that this could be represented using projectors that allow one to rewrite various mereotopological relations between mental objects. What we see is not determined by environmental stimuli but also depends, at least as much, on the way how stimuli are processed.

One approach would demand that being-part-of must be conceived of as being relative to a "parthood-operation":

$$
\forall x \zeta((x<\zeta) \leftrightarrow \exists \widehat{O}(x=\hat{O} \zeta))
$$

where we use the symbol $\zeta$ to account for wholes that are operated upon by $\hat{O}$ to give parts $x$. Experiences are assumed to be constructions relative to the some ("mental") activity. Mathematically, this is best represented by the action of 
operators; the mathematical structure such operators need to conform to is expressed below. A psychological interpretation of this is that perception does not refer to something "given" but to a "resultant." Of course, merely describing something using a particular formalism does not by itself supply an argument, but the construction is intended to emphasize this psychological interpretation. While the "myth of the given" (Sellars 1997) has been much discussed within epistemology, it particularly strikes one as absurd in the field of psychology.

In general, we shall use Greek symbols to refer to objects which are operated on (= "wholes"), whereas Latin symbols denote outcomes of such operations (= "parts"). This distinction is, however, not a principled one, because we assume that parts could themselves be target of a further parthood operation, e.g.,

$$
\hat{O} \hat{O} \zeta=\hat{O} x=y \text {. }
$$

Operators $\hat{O}$ are taken to be associative regarding multiplication and distributive regarding summation:

$$
\hat{O}_{1}\left(\hat{O}_{2} \hat{O}_{3}\right)=\left(\hat{O}_{1} \hat{O}_{2}\right) \hat{O}_{3} \text { and }\left(\hat{O}_{1}+\hat{O}_{2}\right) \zeta=\hat{O}_{1} \zeta+\hat{O}_{2} \zeta
$$

We furthermore assume that every operator $\hat{O}$ could be meaningfully applied to every object $\vartheta$. However, in some (most) cases this will lead to the following result:

$$
\hat{O} \vartheta=0 .
$$

With respect to Eq. (15) this is equivalent to saying that there only exists some ("trivial") object 0 that is part of the object $\vartheta .{ }^{14}$ If this were true for all possible operations,

$$
\forall \widehat{O}(\hat{O} \vartheta=0)
$$

\footnotetext{
${ }^{14}$ Note that one would want to explicitly restrict "connection" as defined in Eq. (7) to objects other than the trivial one.
} 
then the object $\vartheta$ is said to be indivisible (excluding the trivial result). Sometimes, it will be the case that an operation leads to no change in the object that has been operated on:

$$
\hat{O} \vartheta^{\prime}=\vartheta^{\prime}
$$

Then, we will find that:

$$
\hat{O} \hat{O} \ldots \hat{O} \vartheta^{\prime}=\hat{O}^{n} \vartheta^{\prime}=\vartheta^{\prime}
$$

in which case we say that $\vartheta$ is a fixed-point with respect to the operation $\hat{O}$. A particularly interesting class of operators called projectors, $\widehat{P}$, are those which always lead to either a fixed-point or 0 when applied to an object $\zeta$. For such projectors, we find that:

$$
\forall \zeta\left(\hat{P} \hat{P} \zeta=\hat{P} \vartheta^{\prime}=\vartheta^{\prime}=\hat{P} \zeta\right)
$$

In other words, such operators are idempotent ${ }^{15}$ :

$$
\forall \hat{O}\left(\hat{O}=\hat{P} \rightarrow \hat{O}^{n}=\hat{O}\right) .
$$

If we restrict the domain of operators to projectors, we could then characterize "part-of" by specifying the algebra of projectors. We have already discussed idempotency, which accounts for the reflexivity of $\widehat{P}$ with respect to its fixedpoint. There are also other features of the part-whole-relation worth discussing. The most salient issue pertains to transitivity (Simons 1987): If some $x$ is part of $y$ that is part of $z$, is it true that $x$ is also part of $z$ ? It is helpful to write this in the notation from above. Transitivity requires that:

$$
\exists \widehat{P}_{1} \widehat{P}_{2}\left(x=\widehat{P}_{1} y \wedge y=\widehat{P}_{2} z\right) \rightarrow \exists \widehat{P}_{3}\left(x=\widehat{P}_{3} z \wedge \widehat{P}_{3}=\widehat{P}_{1} \widehat{P}_{2}\right)
$$

It can be shown that $\hat{P}_{3}$ is idempotent and thus defines a proper projector iff the operators $\hat{P}_{1}$ and $\hat{P}_{2}$ commute, i.e. iff:

15 The idea of specifying existents in a processual ontology in terms of idempotence has already been discussed by Eddington (1958) and later by Hiley (2011). 


$$
\hat{P}_{1} \hat{P}_{2} z=\hat{P}_{2} \hat{P}_{1} z
$$

This marks a difference between standard accounts of mereology that either acknowledge transitivity to be an axiomatic property of parthood or reject transitivity on a posteriori grounds. In contrast, and according to our calculus, transitivity depends on the way how the corresponding operators behave when executed subsequently on an object $z$.

Similar considerations apply to anti-symmetry, which refers to the idea that if $x$ is part of $y$, then $y$ is part of $x$ only in case $x$ and $y$ are identical:

$$
\forall x y\left(x=\hat{P}_{1} y \wedge y=\hat{P}_{2} x \rightarrow x=y\right) .
$$

Other important operations that we have encountered previously in this article could be re-written in this notation too:

$$
\begin{gathered}
x \circ y \Leftrightarrow \exists \hat{P}_{1} \hat{P}_{2}\left(\hat{P}_{1} x=\hat{P}_{2} y\right), \\
\neg(x \circ y) \Leftrightarrow x<y \Leftrightarrow \forall \hat{P}_{1} \hat{P}_{2}\left(\hat{P}_{1} x \neq \hat{P}_{2} y\right) .
\end{gathered}
$$

The above says that two objects overlap iff there exists a pair of projectors that project to a common object (Fig. 4). Conversely, two objects are disjoint iff they are not overlapping.

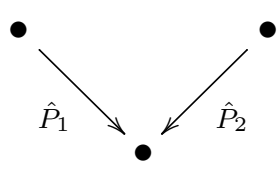

Figure 4: Two objects overlap iff a common projection could be induced by a set of projectors.

Similarly, the notion of mereological connectedness could be redefined in terms of projectors (Fig. 5):

$$
\forall x y\left(C(x, y) \Leftrightarrow \exists z\left(\forall \hat{P}_{z} \exists \hat{P}_{x} \hat{P}_{y}\left(\hat{P}_{z} z=\hat{P}_{x} x \vee \hat{P}_{z} z=\hat{P}_{y} y\right)\right)\right)
$$




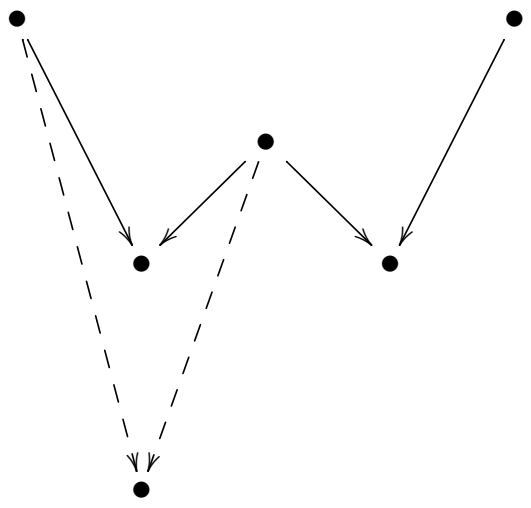

Figure 5: Two objects (top bullets) are connected iff there exists one other object (middle bullet) that overlaps with both of them. Note, that the right part in Eq. (28) demands in addition that every part of said object needs to overlap with one of the two outer objects (indicated by the dashed arrows).

Finally, we could also define boundaries $\xi$ via projectors:

$$
\forall x y\left(B(x, y) \rightarrow \exists \hat{P}_{\xi}\left(\hat{P}_{\xi} \zeta=\xi(\xi \circ x \wedge \xi \circ y)\right)\right) .
$$

Note that $B(x, y)$ by definition implies $C(x, y) \wedge x<y$.-Note also that idempotence - a natural (defining) property of projectors - is crucial to guarantee the fourth Kuratowski-axiom (the closure axiom).

According to the model, the structure of experience could be understood in terms of partitions of phenomenal space. One could further hypothesize that such a partition is induced by endogenous dynamics, which correlates to internal (i.e. not environmentally-driven) forms of information-processing in complex living beings.

Mathematically, projectors have certain well-studied properties (e.g., idempotency, commutativity, their spectral properties...), which makes it suggestive to take the projector-calculus introduced above to represent an operation on phenomenal space that gives objects which one could interpret as "intentional objects". Importantly, this operation need not itself be conscious. Given this, it would seem reasonable to say that experienced objects are "produced" by an unconscious activity. The tri-partite architecture of consciously experienced object-contents, preconscious background and unconscious activity 
is remarkably similar to ideas from psychoanalysis and depth psychology. We are conscious only of a subset of mental processes. In fact, the vast majority of such processes are unconscious (Bargh \& Morsella 2008). Just as we are not aware of the processes of homeostasis, we are most likely not aware of the mental processes that underlie the formation of intentional objects which we consciously experience.

\section{Discussion}

How does the mathematical model developed in section 3 account for the phenomenological characteristics presented in section 2? There, we have argued for three properties that jointly distinguish conscious experience: (i) its embedding in an environment, (ii) the two-way interdependency between local and global states, and (iii) the top-down character that grounds the experience of unified objects. Our model could account for these as follows:

(i) Phenomenal space is defined in terms of a set of quasi-elements which correlate to perception-action couplings between an organism and its environment. This accounts for the environmental embedding of experience. The set of quasi-elements forms the ("preconscious") background for (but does not determine) the individuation of experienced objects.

(ii) The local-global interdependency of experience is mirrored in the mereological and topological structure of phenomenal space. Overlap between elements could explain how preconscious states constitute experience "from the bottom-up" and, at the same time, boundaries make intelligible how experience is globally constrained.

(iii) Restricting the domain of admissible entities to objects that are resultants of operators, allows one to re-write the notions of "overlap" and "boundary" in terms of projectors. The algebra of projectors thus specifies the structure of phenomenal space, and - because structure defines object-content in our model - accounts for the "top-down" (transcendental) character of consciousness.

While it is assumed that (topological) structures of experience, and thus the objects an organism could "see", result from an endogenous (i.e., internal) dynamics defined on phenomenal space; experience is still grounded in the 
interaction between an agent and its world, since the most primitive structural layer of phenomenal spaces (i.e., their pre-topological structure) are taken to correlate to organism-environment couplings.

Yet, there are limits within this model, pointing to a need for further modification. The object-contents of experience do not simply reflect which environmental couplings are actualized or not, but also on the way how different experiences "hang together", for example which experiences are remembered or otherwise related to them, and probably also which experiences are merely possible on that basis. This implies an understanding of consciousness as dynamical entity.

The model we have presented also pertains to empirical debates in the science of consciousness. A conception similar to the most primitive level in our model was put forward by Rosenthal (2005) in his theory of "quality-space." The basic idea is that underlying the explicit representations of awareness, there is a "mental" (= qualitative) space, the elements of which stand in a relation of (dis-) similarity. It was proposed that the (dis-) similarity-structure of quality-space empirically corresponds to the structure of "just-noticeable-differences" in psychophysical experiments. One must not conceive of such spaces in terms of mere aggregates of qualities, which are located in some abstract space, but as structured entities. Our proposal further specifies the possible structure of such a space, namely that of a pre-topology. The distinction between closure and preclosure becomes important here, because it hints at a very natural (but still precise) way of characterizing the transition between the basic elements of quality-space and reportable conscious representations.

Importantly, and unlike Rosenthal's proposal, the basic elements composing quality-space could be assumed as already being phenomenal, given that we do not accept the intuition that phenomenality is strictly bound to conscious report (or "access" more generally). The transition between pre-conscious background and object-representations is thus not proposed here to explain how some states become phenomenal (Rosenthal's own preferred way of arriving at this transition is spelled out in terms of a "higher-order thought" mechanism). In particular, this does not pretend to solve the "hard problem of consciousness" (i.e., explicate the transition from "brain" to "mind"; Chalmers 1995), but it instead specifies the idea that our overt experiences are in fact the result of a layered process of transitions within the mind of an organism.

Such an interpretation also seems compatible with empirical research, e.g., with

Hakwan Lau's (2008) theory of consciousness based on statistical decision theory, 
which he initially interpreted as instance of higher-order thought theory - an interpretation which might be challenged to be more in line with predictive mechanisms (cf. Hohwy 2015). It would be interesting to see whether such predictive processes (more specifically: their Markov-blanket) define a topological closure operation within a structure that relates sensory inputs to motor outputs (Friston 2013; Hoffman et al. 2015, Fields et al. 2018). If this could be shown, this would lend support to identify the mechanisms described by statistical decision theory with the "object-generating" process that was represented in terms of the projector calculus in this work. The road to empirically demonstration seems in principle straight-forward (however, spelling out the technical details might be difficult): While generating predictions from a highdimensional, perhaps neuronally encoded, model could be described in terms of projections (an example is given in the "projective consciousness model" of Rudrauf et al. (2017)); "closure" would correspond to the formation of statistical boundaries on a state space (similar to the example depicted in Fig. 3). That such projections correspond to internal dynamics, as postulated in Section 3.4, is a natural assumption. Less natural is the identification of state spaces on which closure is defined with the pre-conscious background space of our model. Further work is needed to explore this question.

Other well-known debates in consciousness studies pertain to questions such as whether cognitive access and phenomenal consciousness refer to different phenomena (Block 1995, Baars 2002), whether consciousness and attention are empirically distinguishable processes (Tsuchiya \& Koch 2007, Dehaene et al. 2017), or to what degree phenomenal contents are shaped by predictive mechanisms (Hohwy 2013, Clark 2016). Our disregard for an equivocation between phenomenology and consciousness in the access sense seems to force us to endorse Block's distinction of A- and P-consciousness. The issue is subtler than it appears on first sight, though. One could start by noting that the "topdown" processes postulated in our model are likely related to attentional or predictive phenomena, which are to be distinguished from "bottom-up" constitutional processes. Making contents available to global information processing - as postulated in the "global workspace" framework of Baars (2002) - would then correspond to the transition from the preconscious background to awareness. The question then becomes where to locate the phenomenality of experience in this model. Phenomenality could be thought to either emerge from the "top-down" process or already lie in the elements that constitute experience 
"from the bottom up". These options would roughly correspond to Block's notions of A- or P-consciousness respectively. A third, sometimes overlooked, option would postulate instead that a description of these processes is not about spelling out the transition from unconscious (non-phenomenal) to conscious (phenomenal) states, but merely about explicating the transition between different types (or "stages") of conscious experience. This third option is what we opt for, and transitions between stages of awareness are reflected in differing structures defined on phenomenal space (such as pre-topology vs. boundaryinduced topologies in our model).

How could the model be applied empirically? One possibility is to study cases where phenomenology deviates significantly from the normal case, for example in the case of ventral simultanagnosia where subjects are unable to identify two or more objects at the same time (Hoffman 1998), even though they "see" these objects, as inferred from the fact that patients are able to navigate the environment or count scattered objects. Simultanagnosia also comes in a dorsal variant, in which patients are furthermore impaired to do things like counting or reading. A significant conceptual debate that relates to this is whether or not patients should be considered as "being sighted" or "being blind" based on the successful completion of certain tasks (such as counting, reading or navigating). Our model sidesteps these definitional questions by suggesting that different forms of simultanagnosia might reflect different deficiencies in generating the compositional/decompositional structure of phenomenal space (without the problematic reference to "unseen" and "seen" objects). In terms of our model, the deviation from normally functioning consciousness could either stem from a deficiency to project to more than one object, even though phenomenal space is richly composed, or it is a deficiency in phenomenal space's compositional structure, i.e. the pre-conscious background does not form appropriately. Evidence might be gained from looking at the different loci of the brain that play a role in these conditions. In the case of ventral simultanagnosia, patients suffer from damages to the left inferior temporo-occipital region, in the dorsal variant from bi-lateral damages to the parieto-occipital region of the brain. A good test for the model would thus look at whether the neuronal correlates of these deficiencies are structured according to a projective algebra. This could be assessed, for example, by constructing the corresponding functional networks and analyzing them in terms of algebraic topology (a similar, but more ambitious, idea was recently proposed and carried out in part by Reimann et al. 2017), and study how the breakdown of information flow through these networks could best be described. The model predicts that the psychological deficiency observed in 
ventral simultanagnosia should be related to a failure in closure or other notions related to a projector-based algebra, whereas this is not necessarily the case in the dorsal variant.

More generally, a focus not only on which brain area is active or not, but on the rules according to which activation proceeds within the living brain promises to narrow the gap between physiological objects (e.g. neuronal assemblies) and psychological ones (e.g. intentional objects). As a proposal, the (suggested) projective structure of the neuronal dynamics during experimental situations in which sudden transitions in phenomenology are reported (e.g., in paradigms of bi-stable perception or binocular rivalry) could be investigated, once functional networks have been constructed.

Another area in which the proposed model could find application is related to non-standard modes of inference and models of cognition, which presumes, however, that matters of propositional content are related to matters of phenomenology. A geometric re-appreciation of psychology has been suggested recently by Bruza et al. (2015). Regardless of whether it is true that quantum structure needs to be invoked to account for the mathematical structure of psychology (this is an empirical question), we wish to emphasize the idea that the mathematics of psychology might be described in terms of probability theory, only one particular model of which is classical probability calculus (cf. Narens 2015). A possible re-conceptualization of probability theory proceeds via geometry (topology). An algebra of projectors on mental space could be used to represent non-standard probability theories; at the same time, it would encode certain empirical findings in its axioms. For example, (non-)commutativity of projectors could account for contextuality of psychological findings, such as in "question order effects" (Lavrakas 2008, Wang et al. 2014). If two projectors do not commute - i.e., if the result of subsequent executions depends on the order of execution - this explains (or models) the way cognitive systems contextually respond to questions depending on the order they are asked. The assertion here is not that this is merely a question of which kinds of probability theory are adequate for describing phenomena in cognitive science, but that such phenomena are symptoms of a larger underlying mental architecture (namely that of topologically structured phenomenal spaces).

A final note on the "unity" of consciousness, which seems to riddle many approaches into the study of the conscious mind: The leveled (two-step) model presented in this contribution is thought to be able to account for unity in phenomenologically adequate terms, respecting the three "dimensions" of 
environmental situatedness, its (de)compositional and its transcendental aspects. At the same time the model suggests that unity is not a "metaphysically brute" notion but could be further analyzed as generated by a "bottom-up" and "topdown" process. There exists an additional philosophical implication of our approach: What culminated in the hard problem is the pertinent worry that whatever physical process is taking place, it is not intelligible how or why it is related to experience after all. It could as well "happen in the dark." The only way out of this impasse is, in our opinion, to deeply penetrate the question what it is like to consciously experience. One step into this direction is to come up with a formal notational system that accounts for the unifying structure of conscious experience.

The distinction into a "raw" preconscious background and an "emergent" objectcontent conceptually decouples the notions of "presentation" of the environment (assumed to be correlated to the actualization of perception-action couplings), and the notion of a conscious "representation" in terms of intentional objects which depends on the way how the organism partitions its own phenomenal space. In other words, the account sketched above is neither purely representational nor "anti-representational" (Silberstein \& Chemero 2012) and emphasizes that both "representation" and "dynamical couplings" are necessary concepts for the study of experience. ${ }^{16}$ In addition, claims about the nature of "intentionality" are implicitly reflected in this work. The idea that intentional objects are "about" physically real entities (Searle 1983) is rejected in favor of a more refined relation between environmentally embedded "bottom-up" and "topdown" process that give rise to intentional objects. On this view, intentional objects are conceived of in terms of partitions of phenomenal space, and it could be hypothesized that their primary function is to guide adaptive behavior, rather than to inform us about the state of the world "out there." 17

\section{Acknowledgements}

\footnotetext{
${ }^{16}$ In this context, it should also be mentioned that by partitioning phenomenal space and thus constraining the overall state repertoire of an organism, consciousness seemingly "acts back" on its biological substrate - a model for top-down causation which is consistent with the idea that no non-physical efficient causes exist (cf. Atmanspacher \& beim Graben 2007).

17 Note also that this might be in tension with claims appealing to iso- or homomorphism between mental and physical states.
} 
We thank Don Hoffman, Johannes Kleiner, Wanja Wiese and Jeff Yoshimi for helpful comments. We also acknowledge the critical questions and suggestions of two anonymous referees. The work was partly funded by ETH Zürich's Chair of Philosophy and the Swiss National Science Foundation, grant P2EZP1'175109. We report no competing interests. 


\section{References}

Atmanspacher, H. \& beim Graben, P. (2007): Contextual emergence of mental states from neurodynamics. Chaos and Complexity Letters 2, 151-168.

Baars, B. J. (2002): The conscious access hypothesis: origins and recent evidence. Trends in Cognitive Sciences, 6(1): 47-52.

Bargh, J. A., \& Morsella, E. (2008): The unconscious mind. Perspectives on Psychological Scinece 3: 73-79.

Bayne, T. (2010): The Unity of Consciousness, Oxford, Oxford University Press.

Bayne, T. \& Chalmers, D. (2003): What is the unity of consciousness? In A. Cleeremans (ed.): The Unity of Consciousness: Binding, Integration, Dissociation, Oxford, Oxford University Press, pp. $23-58$.

Biederman, I. (1987): Recognition-by-components: A theory of human image understanding, Psychological Review, 94(2):115-142.

Block, N. (1995): On a Confusion about a Function of Consciousness, Behavioral and Brain Sciences, 18: 227-287.

Bruza, P. D., Wang, Z., \& Busemeyer, J. R. (2015): Quantum cognition: a new theoretical approach to psychology. Trends in Cognitive Sciences, 19(7): 383393.

Buzsáki, G., \& Llinas, R. (2017): Space and time in the brain, Science, 358: $482-485$.

Carnap, R. (1967): The Logical Structure of the World. Berkeley, CA, University of California Press.

Casati, R. \& Varzi, A. (1999): Parts and Places, Cambridge, MA, MIT Press.

Chalmers, D. J. (1995): Facing up to the problem of consciousness. Journal of Consciousness Studies, 2(3): 200-219.

Chalmers, D. J. (2014): The Combination Problem for Panpsychism. In G. Brüntrup \& L. Jaskolla (eds.), Panpsychism, New York, Oxford University Press, pp. 1-32.

Chemero, A. (2003): An outline of a theory of affordances. Ecological Psychology, 15(2): 181-195. 
Clark, A. (2016): Surfing Uncertainty: Prediction, Action, and the Embodied Mind, Oxford, Oxford University Press.

Damasio, A. \& Carvalho, G. B. (2013): The nature of feelings: evolutionary and neurobiological origins. Nature Reviews Neuroscience, 14(2): 143-152.

Dehaene, S., Lau, H. \& Kouider, S. (2017): What is consciousness, and could machines have it?, Science, 358: 486-492.

Eddington, A. S. (1958): The Philosophy of Physical Science, Ann Arbor, MI, University of Michigan Press.

Engel, A. K., \& Singer, W. (2001): Temporal binding and the neural correlates of sensory awareness. Trends in Cognitive Sciences, 5(1), 16-25.

Fields, C., Hoffman, D. D., Prakash, C., \& Prentner, R. (2017): Eigenforms, interfaces and holographic encoding. Constructivist Foundations, 12(3): 265291.

Fields, C., Hoffman, D. D., Prakash, C., \& Singh, M. (2018): Conscious agent networks: Formal analysis and application to cognition. Cognitive Systems Research, 47: 186-213.

Friston, K. (2013): Life as we know it, Journal of the Royal Society Interface, 10, 20130475.

Goff, P. (2006). Experiences Don't Sum, Journal of Consciousness Studies, 13(6): 53-61.

Hiley, B. (2011): Process, Distinction, Groupoids and Clifford Algebras: an Alternative View of the Quantum Formalism, in Bob Coecke (ed.), New Structures for Physics, Springer, Heidelberg, pp. 705-752.

Hoffman, D.D. (1998): Visual Intelligence: How We Create What We See. W. W. Norton \& Company.

Hoffman, D.D. Singh, M. \& Prakash, C. (2015): The Interface Theory of Perception, Psychonomic Bulletin \& Review, 22(6): 1480-1506.

Hohwy, J. (2009): The neural correlates of consciousness: New experimental approaches needed? Consciousness and Cognition, 18(2): 428-438.

Hohwy, J. (2013): The Predictive Mind, Oxford, Oxford University Press.

Hohwy, J. (2015): Prediction error minimization, mental and developmental disorder, and statistical theories of consciousness, in R. Gennaro (ed.), 
Disturbed Consciousness: New Essays on Psychopathology and Theories of Consciousness, Cambridge, MA, MIT Press, pp. 293-324.

Kant, I. (1998): Critique of Pure Reason, The Cambridge Edition of the Works of Immanuel Kant, Cambridge, Cambridge University Press.

Koch, C., Massimini, M., Boly, M., \& Tononi, G. (2016): Neural correlates of consciousness: progress and problems. Nature Reviews Neuroscience, 17(5):

$307-321$.

Kosslyn, S. M., Ganis, G. \& Thompson, W. L. (2001): Neural foundations of imagery. Nature Reviews Neuroscience 2(9): 635-642.

Kuratowski, K. (1966): Topology, Volume I, revised ed., Cambridge MA, Academic Press.

Lau, H. (2008): A higher order Bayesian decision theory of consciousness.

Progress in Brain Research 168: 35-48.

Lavrakas, P. J. (ed.) (2008): Question Order Effects, in Encyclopedia of Survey Research Methods, Thousand Oaks, CA, SAGE Publications Ltd, pp. 663 665 .

Marbach, E. (2009): Towards a Formalism for Expressing Structures of Consciousness. In S. Gallagher \& D. Schmicking (eds.): Handbook of Phenomenology and Cognitive Science, Dordrecht, Springer, pp. 57 - 81.

Metzinger, T. (2000): Neural correlates of consciousness: Empirical and conceptual issues. Cambridge (MA), MIT Press.

Narens, L. (2015): Probabilistic Lattices with Applications to Psychology, Advanced Series on Mathematical Psychology (Vol 5), Singapore, World Scientific.

O'Regan, J. K., \& Noë, A. (2001). Acting out our sensory experience, Behavioral and Brain Sciences, 24(5): 1011-1021.

Terekhov, A. V., \& O'Regan, J. K. (2016). Space as an invention of active agents, Frontiers in Robotics and AI, 3(4).

Oizumi, M., Albantakis, L., \& Tononi, G. (2014): From the Phenomenology to the Mechanisms of Consciousness: Integrated Information Theory 3.0. PLoS Computational Biology, 10(5): e1003588.

Poggio, T., \& Edelman, S. (1990). A network that learns to recognize threedimensional objects. Nature, 343(6255): 263-266. 
Reimann, M. W., Nolte, M., Scolamiero, M., Turner, K., Perin, R., Chindemi, G., Dłotko P., Levi, R., Hess, K. and Markram, H: (2017): Cliques of Neurons Bound into Cavities Provide a Missing Link between Structure and Function. Frontiers in Computational Neuroscience, 11, 555.

Revonsuo, A. (1999): Binding and the Phenomenal Unity of Consciousness, Consciousness and Cognition, 8(2): 173-185.

Revonsuo, A. (2006): Inner Presence. Consciousness as Biological Phenomenon. Cambrdige, MA: MIT Press.

Rosenthal, D. M. (2005): Consciousness and Mind, Oxford, Clarendon Press.

Rudrauf, D., Bennequin, D., Granic, I., Landini, G., Friston, K., \& Williford, K. (2017). A mathematical model of embodied consciousness. Journal of

Theoretical Biology, 428: 106-131.

Seager, W. (1995): Consciousness, information and panpsychism, Journal of Consciousness Studies, 2(3): 272-288.

Searle, J. R. (1983): Intentionality: An Essay in the Philosophy of Mind, Cambridge, Cambridge University Press.

Searle, J. R. (2000): Consciousness. Annual Review of Neuroscience, 23: 557578.

Sellars, W. (1997): Empiricism and the Philosophy of Mind, edited by Robert Brandom, Cambridge, MA, Harvard University Press.

Silberstein, M., \& Chemero, A. (2012): Complexity and Extended Phenomenological-Cognitive Systems. Topics in Cognitive Science, 4(1): 35-50.

Sieroka, N. (2015): Leibniz, Husserl, and the Brain, Basingstoke, Palgrave Macmillan.

Simons, P. (1987). Parts. A Study in Ontology, Oxford, Oxford University Press.

Smith, B. (1996): Mereotopology: A theory of parts and boundaries, Data \& Knowledge Engineering, 20: 287 - 303.

Tao, T. (2011): An Introduction to Measure Theory, Providence, RI, American Mathematical Society.

Tononi, G. (2008): Consciousness as Integrated Information: a Provisional Manifesto, The Biological Bulletin 25: 216-242.

Tsuchiya, N., \& Koch, C. (2009): The relationship between consciousness and 
attention. In S. Laureys \& G. Tononi (eds.), The Neurology of Consciousness, New York: Academic Press, pp. 63-78.

Tsuchiya, N., Taguchi, S., \& Saigo, H. (2016): Using category theory to assess the relationship between consciousness and integrated information theory.

Neuroscience Research, 107: 1-7.

von Uexküll, J. (1926): Theoretical Biology, New York, NY, Harcourt, Brace \& Co.

Wang, Z., Solloway, T., Shiffrin, R.M., and Busemeyer, J.R., (2014): Context effects produced by question orders reveal quantum nature of human judgments, Proceedings of the National Academy of Sciences of the USA, 111: 9431-9436

Wang, J., Cherkassky, V. L., \& Just, M. A. (2017): Predicting the brain activation pattern associated with the propositional content of a sentence: Modeling neural representations of events and states. Human Brain Mapping, 38(10): 4865-4881.

Weyl, H. (1988): Riemanns Geometrische Ideen, ihre Auswirkung und ihre Verknüpfung mit der Gruppentheorie, Berlin, Springer.

Wiese, W. (2016): How to solve the problem of phenomenal unity: finding alternatives to the single state conception, Phenomenology and the Cognitive Sciences,16(5): 1-26.

Yoshimi, Jeffrey (2007): Mathematizing Phenomenology, Phenomenology and the Cognitive Sciences, 6(3): 271-291.

Yoshimi, Jeffrey (2011): Phenomenology and Connectionism, Frontiers in Psychology, 2(228). 